\title{
Les variations d'un invariant appliquées aux formes imperfectives du français et de l'anglais
}

\author{
Bardière, Yves \\ Université de Toulon, BABEL EA 2649 \\ y.bardiere@laposte.net
}

\section{Introduction}

La représentation est une des notions clefs de la théorie de G. Guillaume. Très schématiquement, elle marque une étape intermédiaire entre l'expérience et le discours. Elle correspond en effet dans un premier temps à la conversion linguistique de l'expérience et, dans un deuxième temps, à la potentialité de la langue conditionnant le discours. La langue, qui siège dans le plan de la puissance, est le lieu des représentations mentales permanentes et limitées en nombre, autorisant la variété illimitée des emplois momentanés en discours. En clair, la compétence linguistique mais aussi pragmatique (ou communicationnelle) (plan de la puissance) permet au locuteur de s'exprimer en fonction de ses besoins du moment (plan de l'effet). Il s'établit ainsi, en chronologie notionnelle (ou idéelle), une relation de cause à effet entre l'expérience, la langue (ou langage puissanciel) et le discours (ou langage effectif). Langue et discours ressortissent au langage qui procède de l'expérience. En schéma :

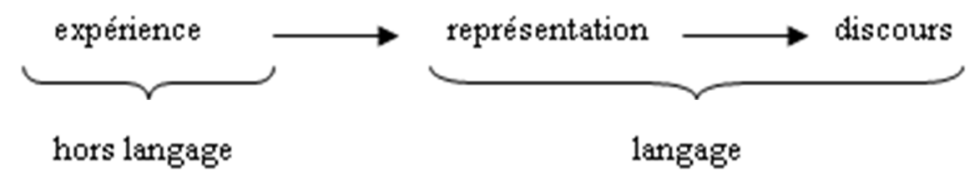

La langue se définit donc comme le lieu de conversion de l'expérience en système de représentations conditionnant à l'endroit du discours. Les différents signifiés d'effet que peut prendre une forme linguistique en discours peuvent être ramenés en langue à un seul signifié de puissance qui les implique tous. Le signifié d'effet doit lui-même être distingué de l'effet de sens qui est la valeur ultime de la forme discursive, modelée par les contraintes co-textuelles (sémantèse du verbe utilisé + environnement textuel e.g. nature du sujet, du complément d'objet, etc.), et contextuelles (situationnelles / pragmatiques). Dans La forme progressive : un point de vue guillaumien, D. O'Kelly propose le schéma suivant :

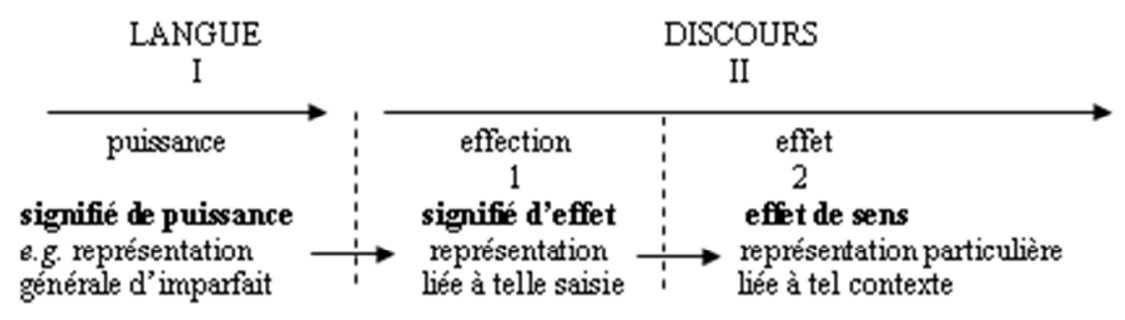

(2000:108)

Je m'intéresserai ici à la fois à la distinction et aux liens qu'il convient d'établir entre expérience, langue et discours, à travers l'analyse des formes grammaticalement imperfectives du français et de l'anglais. Les guillaumiens, à commencer par Guillaume lui-même, ont proposé des adaptations discursives de la 
représentation en langue, correspondant à autant de signifiés d'effet, pour prendre en compte les contraintes de l'expérience. Autrement dit, ils ont introduit des représentations de discours à partir d'une seule et même représentation de langue. Je m'interrogerai donc sur cette adaptation du signifié de puissance: quelle avancée apporte-t-elle dans l'analyse ? Faut-il faire varier l'invariant pour une meilleure appréhension des phénomènes linguistiques ? Pour essayer d'apporter quelques éléments de réponse, j'examinerai plus particulièrement trois cas de figure : 1) l'imparfait narratif 2) l'imparfait modal (à valeur contrefactuelle) 3) la représentation de $\mathrm{BE}+-\mathrm{ING}$ dans les énoncés à sens futur. Ces trois emplois ont pour caractéristique commune de faire intervenir ce que je nomme «le principe de variabilité inversement proportionnelle d' $\omega$ et $\alpha \gg$. Afin de mieux cerner les enjeux de la question, voici tout d'abord un bref rappel sur les « formes imperfectives » du français et de l'anglais.

\section{La représentation invariante des formes imperfectives et perfective du français et de l'anglais}

\subsection{L'imparfait et le passé simple français}

Je considérerai le trait [+sécant] comme l'invariant aspectuel de l'imparfait. C'est celui qui a le plus de force explicative, les autres traits aspectuels ([+ intérieur], [+ statique], [- relief]), etc. pouvant être dérivés de cette valeur fondamentale. Le procès exprimé à l'imparfait est perçu de l'intérieur, ce qui permet de le scinder en deux parties, une partie déjà réalisée ou accomplie $(\omega)$ et une autre virtuelle ou non accomplie $(\alpha)$. En schéma :

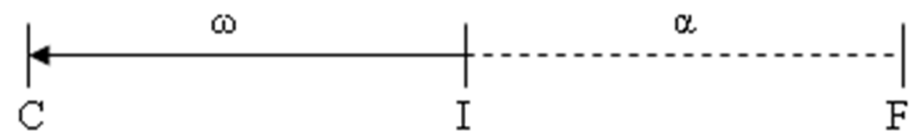

I (Instant de saisie) réfère à l'instant que choisit l'observateur-énonciateur ( $v$. note viii) pour saisir le procès dans son déroulement interne. L'instant de saisie est fréquemment explicité par un événement au passé simple qui vient s'insérer dans le déroulement interne du procès à l'imparfait. C'est l'«Inzidenzschema » (schéma d'incidence) de Pollak (1961). I remplit en fait quatre offices :

1) Il exerce la fonction d'instant séparateur.

2) Il est le point de passage du temps intérieur ou point de résolution d' $\alpha$ en $\omega$.

3) Il est le point de localisation du sujet de l'énoncé dans l'immanence de l'événement.

4) Il est le point d'observation par le sujet de l'énonciation (ou son image). Celui-ci se transporte au cœur du procès et l'observe de l'intérieur (v. M.-F. Delport et J.-C. Chevalier 1995a : 150 ; v. également note viii)

Certains linguistes remettent en cause la valeur sécante induite par l'imparfait. C'est le cas de Molendijk et Vet qui arguent du fait que dans un énoncé tel que Quand Pierre atteignit le sommet de la montagne, une heure sonnait au clocher, l'atteinte du sommet de la montagne et l'unique coup de cloche offrent l'un comme l'autre une image ponctuelle de l'événement évoqué. Dans Grammaire critique du français, M. Wilmet rétorque par cette citation de Joe Larochette :

(1980, II, 194) [Il sonnait une heure quand nous nous mîmes à table]. Dans la réalité, le début du premier mouvement d'un des convives a-t-il eu lieu alors que résonnait déjà le coup de cloche? Cela n'est pas pertinent. Ce qui est pertinent, c'est que l'opposition de l'imparfait et du passé simple représente les choses comme si elles s'étaient passées ainsi (1998: 406 ; c'est moi qui souligne).

La réponse de $\mathrm{M}$. Wilmet est tout à fait convaincante mais elle gagnerait à désigner avec plus de netteté, l'origine du problème, à savoir la confusion entre la représentation de la forme en langue et la réalité du monde phénoménal.

Autre exemple de confusion entre référent mental et référent expérientiel: selon Riegel et al., l'interruption du procès signifierait l'occultation de sa partie non accomplie : 


\begin{abstract}
Son aspect analytique permet d'introduire une rupture exprimée par le passé simple : Une nuit qu'il dormait, il crut entendre quelqu'un l'appeler (Flaubert) - Depuis une heure, il avançait ainsi, lorsque sur la gauche, à deux kilomètres de Montsou, il aperçut des feux rouges, trois brasiers brûlant en plein air, et comme suspendus (Zola). Le passé simple vient interrompre le procès à l'imparfait en dissociant ses deux parties : la partie initiale est réalisée, la partie virtuelle est annihilée (1999: 306 ; c'est moi qui souligne).
\end{abstract}

Le procès serait interrompu et la partie $\alpha$ (non accomplie) annihilée. Pourtant, dans les exemples choisis, crut entendre n'implique pas nécessairement l'interruption du processus dormir ou, dans la citation de Zola, la vision des feux rouges n'entrave pas obligatoirement la progression du personnage. De même, dans Il lisait depuis plus de deux heures lorsqu'il fut terrassé par une apoplexie foudroyante, le temps de lecture se trouve certes tristement écourté, mais ce n'est pas le passé simple qui abrège le processus, c'est l'apoplexie! Autrement dit le passé simple n'interrompt pas la résolution de l'inaccompli en accompli du procès à l'imparfait, la partie virtuelle n'est pas « annihilée ». L'approche de Riegel et al. superpose en définitive les deux plans de l'expérience et de la représentation de la forme verbale en langue. Le passé simple marque un repère temporel qui correspond à l'instant de saisie sécante du procès à l'imparfait sans prendre en compte la continuation ou l'interruption de l'événement au-delà de ce point dans le monde phénoménal.

On notera enfin que, dans la représentation graphique, le vecteur orienté vers la gauche indique que le temps événementiel fuit du futur vers le passé lors de son passage en I (Instant de saisie), avec résolution de l'inaccompli en accompli ${ }^{1}$. Soit par rapport au mouvement fondamental qui sous-tend tout événement et selon lequel on voit celui-ci se développer de son début à sa fin :

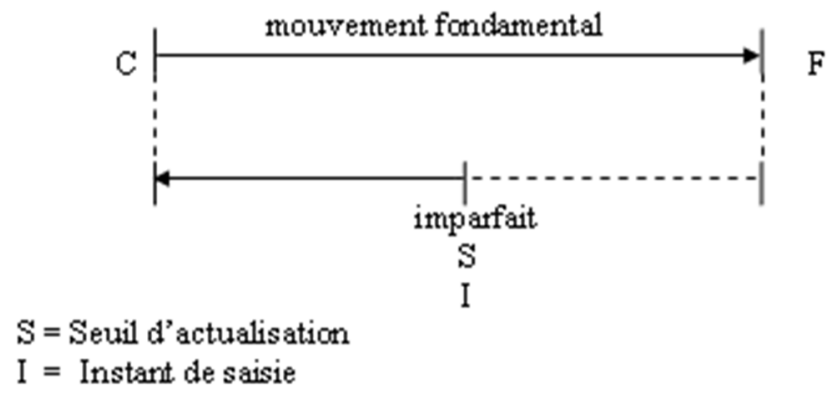

Le passé simple offre, quant à lui, l'image d'un événement se déroulant intégralement depuis son commencement $(\mathrm{C})$ à sa fin $(\mathrm{F})$. Il épouse dans ce cas le cinétisme fondamental:

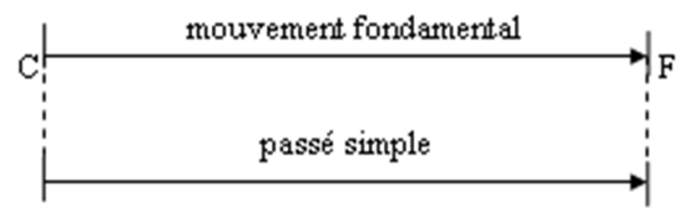

Dans la réalité d'expérience, il arrive que seul le début de l'événement soit effectivement actualisé, le reste étant laissé en accomplissement prospectif. L'énoncé Il alla à la fenêtre peut en effet s'interpréter comme un procès effectif (fenêtre atteinte) ou inceptif (il se dirigea vers la fenêtre). Ces différents signifiés d'effet sont subsumés dans le signifié de puissance de la forme de langue. Il est là encore fondamental de distinguer les plans de l'expérience et de la représentation en langue. 


\subsection{Le prétérit en BE+-ING et le prétérit simple anglais}

La subduction ésotérique ${ }^{2}$ ou dématérialisation sémantique de $\mathrm{BE}$ fait passer le verbe lexical plein $(\mathrm{BE}=$ exister) à la copule (morphème translatif permettant le passage du plan nominal au plan verbal) et enfin à l'auxiliaire. Celui-ci correspond à la dématérialisation la plus engagée, mais la matière qu'emporte l'auxiliaire n'est jamais réduite à zéro. L'auxiliaire joue en effet un double rôle : d'une part il localise l'événement dans le temps d'univers d'autre part il situe le sujet de l'énoncé dans l'événement, i.e. dans son intériorité, son immanence La forme quasi-nominale évoque elle aussi l'intériorité de l'événement « ou plus précisément, la résolution du non accompli en accompli » (A. Joly \& D. O’Kelly $1990: 259$ ).

De cette intériorité doublement suggérée par BE et ING, un seul instant I est actualisé, ce qui a pour effet de couper l'événement en deux parties, une partie déjà accomplie symbolisée par $\omega$ et une partie à accomplir symbolisée par $\alpha$.: "la forme dite progressive est une forme verbale par nature sécante d'aspect imperfectif» (op. cit. : 265), qui invite à représenter l'événement désigné par le verbe en termes de « déjà » et «pas encore ». Par exemple, dans le schéma ci-dessous, le déroulement de l'événement se trouve interrompu en $\mathrm{i}_{4}$. De part et d'autre de cette saisie, située quelque part dans l'intériorité de l'événement, entre son commencement $\mathrm{C}$ et sa fin $\mathrm{F}$, les instants précédents sont vus rétrospectivement comme des supports d'accompli, le reste de l'événement comme du non accompli perspectif, représenté par une ligne brisée sur la figure :
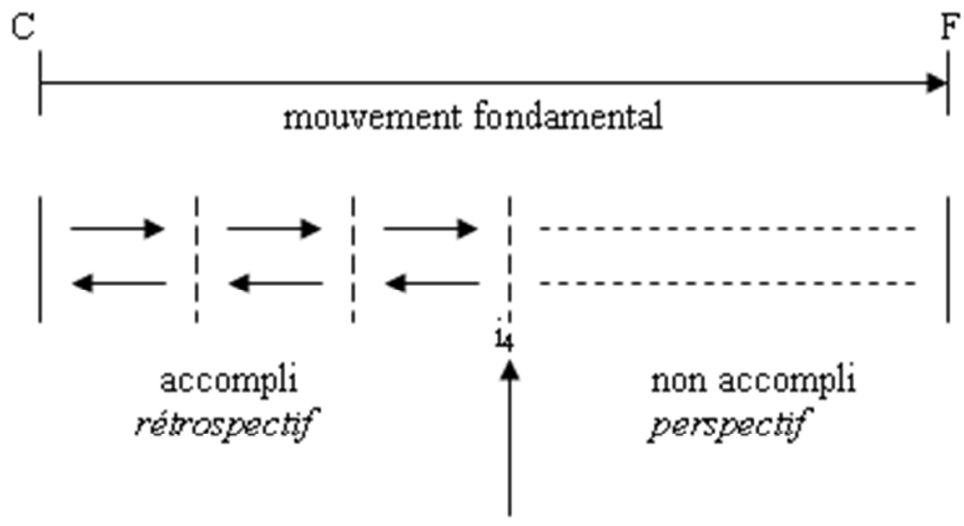

instant de saisie de l'événement

Au mode quasi-nominal, le temps d'univers ou temps porteur est descendant, les événements viennent du futur, passent par le présent et fuient vers le passé. Le temps d'événement, qui interpole le temps d'univers, épouse la même orientation, la résolution de l'inaccompli en accompli imprimant un mouvement descendant. Aussi l'utilisation du terme perspectif, impliquant a priori un cinétisme temporel ascendant, peut elle paraître inappropriée. «Cinétisme descendant » et « vue perspective » ne sont en réalité pas aussi incompatibles qu'il y paraît à première vue. Il est en effet possible de jeter un regard prospectif sur du temps arrivant, c'est-à-dire sur la partie en cours de résolution de l'inaccompli en accompli.

La forme simple ne présente pas, quant à elle, cette vision anisogène $e^{3}$ de l'événement mais montre celuici se dérouler de $\mathrm{C}$ (Commencement) à $\mathrm{F}$ (Fin) intégralement et dans le sens $\mathrm{C} \rightarrow \mathrm{F}$ (orientation vers le futur). Il est cependant capital de distinguer le plan de la puissance (en langue) du plan de l'effet (en discours). La perfectivité de langue du prétérit simple se prête en discours à trois saisies cardinales que, par commodité terminologique, je nommerai "perfectivité intégrale», "perfectivité inchoative» et "perfectivité médiane». Intégrale, inchoative et médiane ne réfèrent qu'à la partie effective de l'événement. Ce qui, dans la réalisation discursive n'est pas effectif est puissanciel. Soit les exemples et les schémas suivants où la partie effective + la partie puissancielle = l'entier de l'événement :

- la perfectivité intégrale, qui offre une vue effective de la totalité de l'événement :

She went to the window (fenêtre atteinte). 


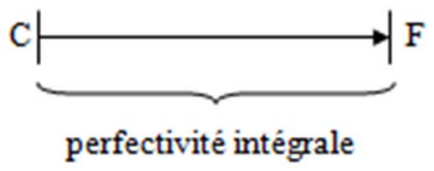

- La perfectivité inchoative, où seul le premier instant saisi de manière effective. Tout le reste est puissance :

She went to the window (Elle se dirigea vers la fenêtre).

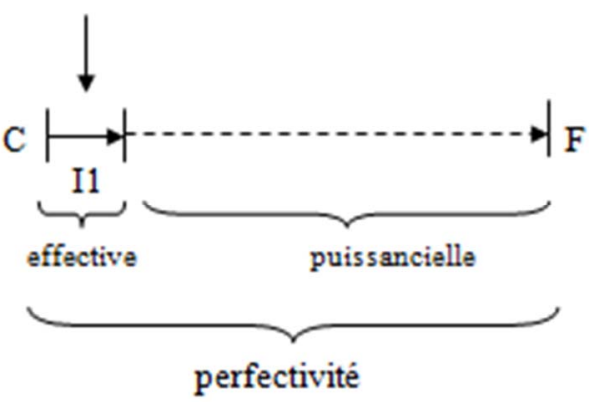

Dans ces deux premiers cas, le prétérit simple donne l'image d'un événement parcouru intégralement du début à la fin mais il est saisi effectivement ou puissanciellement en $\mathrm{F}$, selon que le sujet, dans la réalité exprérientielle atteint ou non la fenêtre. Le passé simple présente des propriétés de langue et de discours analogues (v. supra, § 1.1.). Il ne saurait toutefois être considéré comme le calque du prétérit simple. Les formes de l'anglais et du français présentent des différences importantes, le prétérit simple (forme perfective) pouvant se traduire par l'imparfait (forme imperfective).

- la perfectivité médiane, où tout autre instant ou série d'instants, compris entre le début et la fín du procès, sont effectivement actualisés :

Jim, as I've told you, accompanied me on the first stage of my journey back to the world he had renounced, and the way at times seemed to lead through the very heart of untouched wilderness. The empty reaches sparkled under the high sun; between the high walls of vegetation the heat drowsed upon the water, and the boat, impelled vigorously, cut her way through the air that seemed to have settled dense and warm under the shelter of lofty trees. (Lord Jim, pp. 249-250)

Comme je vous l'ai dit, Jim m'accompagna dans la première étape de mon voyage de retour vers ce monde auquel il avait renoncé, et notre itinéraire semblait par moments nous transporter au cour même d'une jungle inviolée. Les portions dégagées de la rivière étincelaient sous le soleil déjà haut; la chaleur sommeillait sur l'eau; et le bateau, mû par des bras vigoureux, fendait l'épaisseur de l'air sous l'immense dôme des arbres qui en augmentait la chaleur suffocante. (Traduction d'Odette Lamolle, pp. 359-361)

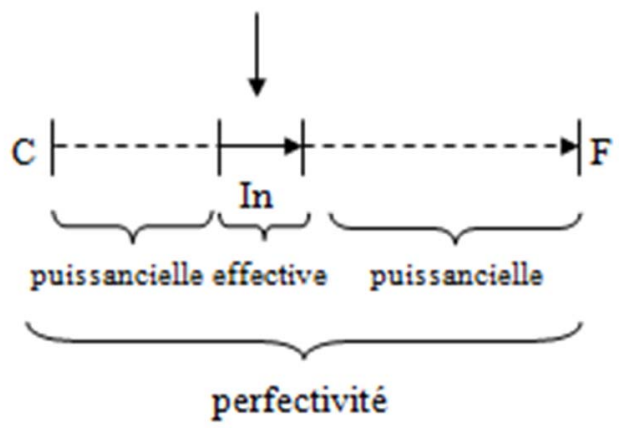


C'est le seul cas où le prétérit simple entre en concurrence avec le prétérit en BE+-ING pour traduire l'imparfait français. A une forme imperfective du français peut correspondre en effet une forme imperfective mais aussi perfective de l'anglais. C'est là que réside l'une des difficultés les plus déconcertantes pour le traducteur du français vers l'anglais. L'examen comparatif des cas répertoriés ici permet d'aboutir au tableau récapitulatif suivant :

\begin{tabular}{|c|l|l|l|l|}
\hline \multirow{2}{*}{ ANGLAIS } & \multirow{2}{*}{ LANGUE } & \multicolumn{2}{|c|}{ DISCOURS } & \multicolumn{1}{|c|}{ FRANÇAIS } \\
\hline \multirow{2}{*}{ prétérit simple } & \multirow{2}{*}{ perfectivité } & intégrale & & PS \\
\cline { 3 - 5 } & & \multirow{2}{*}{ partielle } & transitive & IMP \\
\cline { 4 - 5 } & & & inchoative & PS \\
\hline
\end{tabular}

Les critères susceptibles de favoriser une lecture intégrale, inchoative ou médiane de la perfectivité grammaticale du prétérit simple et donc, in fine, sa traduction par le passé simple ou l'imparfait sont extrêmement nombreux et variés : extension temporelle du champ d'application du procès, deixis ad occulos, traits [+/- dynamiques] du procès et [+/- agentif] du sujet, procès perçus en simultanéité ou consécutivité, agencement typographique du texte, etc. Je ne peux, dans le cadre d'une étude qui s'interroge prioritairement sur les variations de l'invariant aspectuel des formes imperfectives plutôt que perfectives du français et de l'anglais m'étendre davantage sur le sujet ${ }^{4}$.

\section{Les variations de l'invariant appliquées à l'imparfait}

\subsection{L'imparfait narratif}

\subsubsection{La double saisie aspectuelle de l'imparfait : perfectivité vs imperfectivité}

Sans doute le critère le plus intuitif de reconnaissance d'un imparfait narratif est la possibilité d'employer à sa place un passé simple orthonymiquement ${ }^{5}$ attendu, sans nuire à la cohérence de l'énoncé ni modifier l'interprétation chronologique des événements. Cette proximité a incité certains linguistes à considérer qu'il existe deux types d'imparfait, un imparfait perfectif et un imparfait imperfectif. Par exemple, Tasmowski-De Ryck (1985) définit l'imparfait narratif et le passé simple par des traits identiques, à savoir [+globalité] / [+progression], par opposition à l'imparfait standard caractérisé, quant à lui, par les traits [-globalité] / [-progression]. De même, L. Gosselin considère que «l'aspect global (aoristique) constitue [...] la différence spécifique entre l'IP standard (inaccompli) et l'IP narratif, qui s'avère être compatible avec tout un ensemble de marqueurs impliquant l'aspect global et qui, de ce fait, excluent l'IP standard » (1999: 22). D. Maingueneau va plus loin dans la mesure où cette double valeur aspectuelle concernerait tous les temps de l'indicatif et pas seulement l'imparfait. Les traits [+perfectif] et [-perfectif] seraient inscrits dès le départ dans les gènes de chacune de ces formes et seul l'un d'entre eux serait activé en discours (1994: 81).

Cette approche laisse un arrière-goût de compromission théorique visant concilier l'inconciliable. Comment deux signifiés aspectuels aussi radicalement antinomiques peuvent-ils coexister au sein d'une seule et même forme verbale? Le paradoxe peut être résolu en affirmant, au contraire, que l'imparfait présente une même représentation aspectuelle imperfective de son image-temps, quels que soient ses emplois en discours. L'analyse retrouve ainsi toute sa cohérence par l'attribution d'un seul invariant qui, ici plus qu'ailleurs, devient l'élément explicateur de cette saveur si particulière de l'imparfait narratif. 


\subsubsection{Les variations de l'invariant imperfectif : incidence vs décadence}

Si les psychomécaniciens veillent à distinguer scrupuleusement les trois étapes de la chronologie idéelle, il arrive cependant que la représentation, phase intermédiaire entre l'expérience et l'expression, puisse faire, de leur part, à commencer par G. Guillaume lui-même, l'objet d'une adaptation, pour ne pas dire d'une déformation, dictée par des contraintes d'ordre extralinguistique ou discursif.

En 1929, dans Temps et Verbe, G. Guillaume commentait en ces termes l'emploi des « imparfaits stylistiques $\gg$ :

[...] les imparfaits obtenus par ce moyen sont toujours susceptibles d'être remplacés par le parfait défini sans qu'il s'ensuive autre chose que le sacrifice d'une nuance. Autrement dit ces imparfaits nous mettent en présence d'une forme dépourvue de valeur grammaticale stable, mais qui n'en possède pas moins une valeur caractérisée, d'où il ressort que, grammaticalement interchangeables avec le parfait défini, ces imparfaits sont loin, cependant d'en être l'équivalent expressif (p. 66).

La citation se borne à un simple constat, en reste au niveau de l'effet de sens, sans faire la démonstration linguistique de la nuance en question. La pensée de l'auteur s'affine et se précise dans le premier recueil des Leçons de linguistique de 1946 :

L'imparfait, partout et toujours, satisfait à la condition de langue: accompli décadent + accomplissement incident $=1$. Mais en discours, l'accompli (décadent) peut aussi passer du positif indiscuté à un positif discuté, avoisinant zéro d'aussi près que possible tout en restant positif. [...] La variation n'est pas une variation formelle qualitative, mais une variation de quantité, de proportion des quantités relatives retenues. La forme, obtenue en système, en langue, non en discours, est invariante (pp. 103-104).

Cette analyse est reprise en des termes plus explicites encore par A. Joly et A. Boone. L'imparfait peut présenter en discours des valeurs fort différentes, allant parfois jusqu'à la contradiction, telles que l'actualisation $v s$ la virtualisation du procès ou encore, comme ici, la "perfectivité » ${ }^{6} v s$ l'imperfectivité. Ces valeurs d'emploi s'expliquent par la variabilité de la proportion d'accompli dans le déroulement interne du procès. Tout dépend du positionnement de la saisie sécante, délimitant à l'intérieur du temps d'événement, la part respective d'accompli et d'inaccompli :

Dans Pierre chantait, comme dans tout imparfait de type courant, l'accompli rétrospectif est équilibré par l'accomplissement prospectif Mais si l'accompli tend vers zéro au bénéfice de l'accomplissement, on obtient des imparfaits "spéciaux », extrêmement proches par leur contenu du passé simple et sujets à alterner expressivement avec ce dernier (imparfait "pittoresque » des grammairiens). Comparez Il y a trois mois un homme bien mis et de grandes manières se PRESENTAIT chez un bijoutier (imparfait $=$ décadence au voisinage immédiat de la nullité + incidence au voisinage immédiat de la totalité) à Il y a trois mois un homme bien mis et de grandes manières se PRESENTA chez un bijoutier (passé simple = décadence nulle + incidence totale) (A. Joly et A. Boone 1996: 1314).

En schéma :

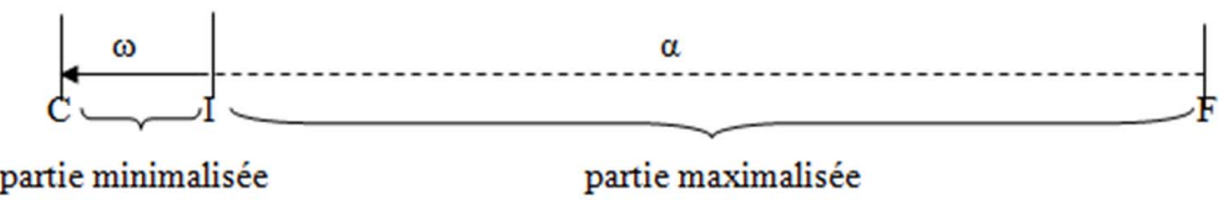

\subsubsection{Remise en cause de l'approche théorique}

Je m'interrogerai ici sur cette déformation discursive de l'image-temps. Un détour s'impose par la prise en compte de l'aspect lexical et plus particulièrement de l'interaction entre aspect lexical et aspect grammatical. Se présenter est perfectif sur le plan lexical. Or les meilleurs candidats à l'imparfait narratif sont précisément les processus lexicalement perfectifs. De plus, l'aspect lexical est une proposée et l'aspect grammatical est une transformée. La matière lexicale, travaillée de l'intérieur, est transformée, à 
des degrés divers, lors de son actualisation en discours, par l'aspect grammatical ainsi que, de manière générale, tout élément du cotexte et du contexte susceptible d'influer sur la sémantèse verbale. Dans Il y a trois mois un homme bien mis et de grandes manières se présentait chez un bijoutier, se présenter, procès lexicalement perfectif, est simplement « dilaté » par l'imparfait.

Cette dilatation du seuil télique permet de distinguer le terminus a quo du terminus ad quem et donc d'effectuer une saisie sécante dans l'intériorité événementielle. La représentation de l'imparfait appliquée à un processus lexicalement perfectif devient alors similaire à celle de l'imparfait utilisée avec un processus lexicalement imperfectif, autrement dit «l'accompli rétrospectif est équilibré par l'accomplissement prospectif » (op. cit.).

Deux attitudes sont alors possibles : soit on considère que le procès se déroule dans son intégralité, auquel cas il est perfectif, soit on considère que l'imparfait montre l'événement comme s'il était en cours de résolution, indépendamment de la pression co-textuelle exercée par le complément adverbial et la valeur perfective de l'aspect lexical (se présenter), auquel cas l'imparfait conserve une représentation aspectuelle invariante. Le principe de variabilité d' $\alpha$ et $\omega$ me semble relever, du moins en ce qui concerne l'imparfait narratif, d'un compromis visant à concilier les exigences contradictoires de la langue (valeur imperfective) et du discours (effet « perfectivant»), sans véritablement satisfaire aux deux. Il ne saurait, à mon sens, être fait appel à une décadence quasi nulle dans le seul but de sauvegarder la représentation imperfective. L'imparfait narratif me parait au contraire tirer toute sa mesure et sa puissance d'une imperfectivité invariante, même en discours. C'est de ce conflit entre la perfectivité «co-textuelle » (i.e. l'effet de sens «perfectif», véhiculé par le cotexte) et l'imperfectivité aspectuelle portée par l'imparfait, que jaillit la valeur «stylistique ».

\subsection{De la valeur aspectuelle à la valeur modale}

Dans un article au titre accrocheur, Les variations d'un "invariant»: approche morphogénétique de l'imparfait français, A. Joly montre que la valeur fondamentale de l'imparfait est d'être une «proposée de formes ", en d'autres termes, elle est porteuse de variabilité et de transformations. Selon l'auteur, $\omega$ est réduit à zéro $(\omega=\varnothing)$ et $\alpha$ occupe l'intégralité de l'événement $(\alpha=1)$ dans des énoncés du type Un instant après, le train déraillait (interprétation contrefactuelle) ou Sans vous je tombais ${ }^{7}$.

La démarche présente un grand intérêt car elle distingue, via l'aspect, l'imparfait narratif, où l'accompli décadent passe «du positif indiscuté à un positif discuté, avoisinant zéro d'aussi près que possible tout en restant positif» (v. supra, § 2.1.2.; c'est moi qui souligne) de l'imparfait modal, où l'accompli décadent se réduit à zéro au profit de l'inaccompli incident qui occupe alors la totalité du temps événementiel. Cette double représentation de l'image-temps permet de rendre compte de la valeur sémantique diamétralement opposée que peut prendre une forme verbale au sein d'un même énoncé. Ainsi, Un instant après, le train déraillait signifiera que le train a déraillé ou au contraire n'a pas déraillé selon la lecture narrative ou modale qui est faite de l'imparfait.

Mais la configuration aspectuelle de l'imparfait modal soulève une nouvelle difficulté théorique : si l'on considère que « l'imparfait est une forme verbale qui appartient conjointement aux niveaux d'incidence et de décadence » (A. Boone \& A. Joly $1996: 223$ ), autrement dit, que cette configuration anisogène ( $v$. note iii) de l'image-temps constitue l'invariant aspectuel de l'imparfait, comment faire exister la variable $\omega$, quand celle-ci se trouve réduit à $\varnothing$ ?

Le concept de « décadence transcendante » mis en avant par A. Boone apporte, du moins à première vue, une solution originale. La décadence transcendante consiste en effet à accorder une existence à $\omega$ dans l'extériorité d'avant de l'événement :

Il arrive aussi que pour des raisons stylistiques la décadence, au lieu de s'annuler purement et simplement (ce qui entraînerait l'emploi du passé simple), soit transférée à l'extérieur du verbe ( décadence transcendante »). Elle reste ainsi positive dans Un instant après le train déraillait (= «dérailla », l'« instant» a eu lieu), mais devient négative dans Un instant après le train déraillait (= «aurait déraillé », l'« instant» n'a pas eu lieu). Ce dernier 
imparfait, entièrement perspectif, est habile à signifier non pas ce qui a eu lieu, mais ce qui a été évité de justesse. (op. cit. : 224)

La solution est intéressante mais elle fait surgir à son tour deux nouvelles questions. D'une part, pour analyser la configuration aspectuelle d'une forme immanente, telle que l'imparfait, elle contraint l'analyse à quitter l'immanence événementielle, pour se situer dans l'extériorité de l'événement. D'autre part - ceci étant la conséquence de cela - placer $\omega$ dans l'extériorité d'avant de l'événement, revient à octroyer à $\omega$ une valeur négative. Le seul moyen de conférer à $\omega$ la valeur $\varnothing$ - qui n'est d'ailleurs, sur le plan mathématique, ni positif ni négatif - serait de considérer que $\omega$ se confond avec le seuil qui sépare l'extériorité de l'intériorité de l'événement. Il ne se situerait alors ni dans la transcendance d'avant ni bien sûr dans l'immanence de l'événement ... et sombrerait dans l'inexistence - ce qui nous ramène à notre problématique de départ.

Est-il donc nécessaire, dans une théorie de la représentation, de réduire la part d'accompli à la quasinullité ou à la nullité ou a fortiori de faire appel au concept de décadence transcendante ? Faut-il recourir au principe de déformabilité discursive de l'invariant de langue ? Ne suffit-il pas de (re)présenter le sujet observateur ( $v$. note viii) comme s'il était déjà engagé dans le processus événementiel, même si dans le monde réel, le procès n'est envisagé que de manière purement virtuelle?

\section{Les variations de l'invariant appliquées à BE+-ING}

\subsection{Principaux signifiés d'effet}

\subsubsection{Présentation de la théorie}

Les principes qui viennent d'être énoncés au sujet de l'imparfait s'appliquent mutatis mutandis à la forme en BE+-ING, imperfectivité oblige. La saisie en I (Instant d'actualité du temps d'événement est comparable à un curseur déplaçable entre $\mathrm{C}$ et $\mathrm{F}$, pouvant faire tendre $\alpha$ (partie non accomplie) et $\omega$ (partie accomplie) vers la quasi-intégralité $(\rightarrow$ Int) et la quasi nullité $(\rightarrow 0)$, ou inversement, sans pour autant parvenir à l'intégralité ou la nullité absolues.

Dans Grammaire systématique de l'anglais (p. 268), A. Joly et D. O'Kelly illustrent les principaux signifiés d'effet de $\mathrm{BE}+\mathrm{ING}$ à travers trois exemples qui manifestent ce qu'ils appellent « trois saisies cardinales $»$ :

(1) I'm leaving tomorrow.

(2) When the telephone rang, I was working.

(3) I'm passing (jeu de cartes).

En schéma :

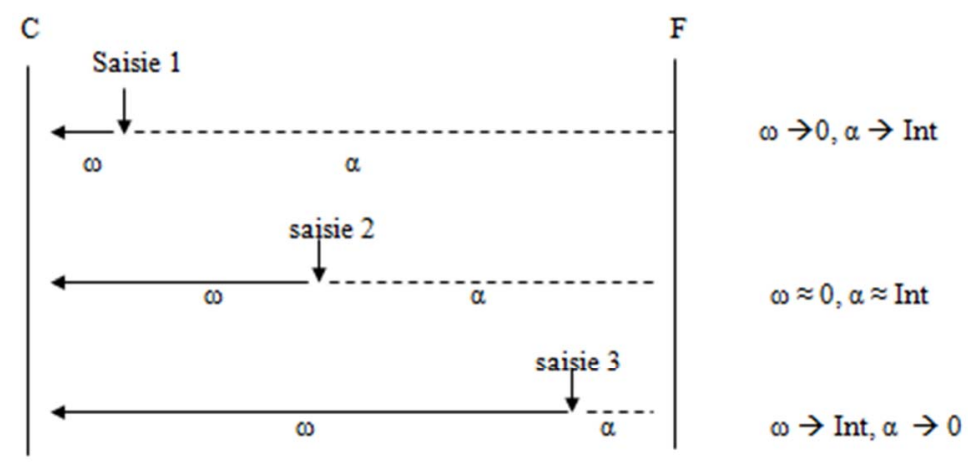

$(1990: 268)$ 
Les auteurs justifient comme suit ces trois positions-types :

1) au plus près de $C$, la limite de commencement : c'est le cas lorsque la variable $\omega$ tend vers zéro. En d'autres termes, lorsque la position d'accompli - l'impression de « déjà » n'existe que pro forma, alors que le reste de l'événement, la portion de non accompli $\alpha$, est vu tout en perspective :

I'm leaving tomorrow.

qui, nuances à part, équivaut à I leave tomorrow.

2) Au plus près de $F$, la limite de fin: situation inverse, obtenue lorsque la variable $\omega$ d'accompli occupe la quasi-intégralité de l'événement, la variable $\alpha$, de non accompli, étant alors réduite, pro forma, au tout dernier instant. C'est l'effet produit lorsqu'au jeu de carte on passe son tour en déclarant :

I'm passing.

qui, toujours compte non tenu des nuances, pourrait se dire : I pass, forme verbale qui oblige à parcourir perfectivement la totalité de l'événement.

3) Enfin toute position intermédiaire où les deux variables, $\omega$ et $\alpha$ peuvent prendre toutes les valeurs positives possibles. Exemple-type :

When the telephone rang, I was working. (op. cit. : 267-268)

La figure n'indique que trois positions cardinales de saisie de l'événement, mais la flèche verticale, symbolisant l'instant de saisie, est mobile, et le nombre de positions, référant à autant de signifiés discursifs, est théoriquement illimité. Ces trois saisies cardinales correspondent à des signifiés d'effet « calculable[s] donc généralement prédictible[s] » (op. cit.) en discours.

Ces variations potentielles sont inscrites dès le départ, dans le signifié de puissance. D. O'Kelly a forgé le concept de «potentialité aspectuelle» pour rendre compte de la variabilité discursive d' $\alpha$ et $\omega$ et expliquer notamment le rapport syntagmatique de comptabilité ou d'incompatibilité entre plusieurs procès dans leurs réalisations discursives ( $v$. A. Joly et D. O’Kelly $1990: 140$ ).

\subsubsection{Mise en question de l'approche théorique}

On se demandera, là encore, pourquoi dans les énoncés performatifs du type I'm passing, $\omega$ occuperait la quasi-totalité de l'intériorité événementielle au détriment d' $\alpha$, réduit, pro forma, au tout dernier instant. Est-ce dû au fait que l'événement n'est pas sitôt énoncé qu'il est déjà accompli ? A quoi correspond alors cette variable $\alpha$ en représentation?

De même, pourquoi $\omega$ se trouverait-il réduit à la quasi-nullité dans les énoncés en BE+-ING à sens futur, tels que My train's leaving at five, c'est-à-dire des événements qui, sur le plan pragmatique, n'ont pas encore commencé et ne présentent donc aucune part d'accompli ? A quoi correspond cette part minimale d'accompli en représentation? L'irruption de la réalité d'expérience dans une théorie de la représentation revient à superposer le plan temporel ou temps de l'événement (i.e. sa localisation dans la chronologie expérientielle) sur le plan aspectuel ou temps d'événement (i.e. le temps intérieur). On exige du temps d'événement qu'il intègre les fluctuations possibles du temps de l'événement. Autrement dit, on fait intervenir ici le temps d'expérience dans une représentation de l'aspect.

La réduction d' $\alpha$ et $\omega$ me paraît, une fois de plus, une sorte de compromis, pour ne pas dire compromission, entre les impératifs de l'expérience où l'événement est entièrement virtuel et ceux de la représentation d'une forme imperfective qui impose l'image d'un procès sécant. De deux choses l'une : soit la part d'inaccompli est considérée comme inexistante, auquel cas elle n'a pas à être représentée, soit la représentation ne tient pas compte de cette contrainte expérientielle auquel cas le quantum accordé à $\alpha$ et $\omega$ devient sans importance. C'est, pour ma part, cette deuxième solution que je préconiserai.

On pourrait s'attendre à ce que le principe de variabilité d' $\alpha$ et $\omega$, appliqué à des événements futurs, soit également applicable à des événements passés, le futur et le passé marquant, l'un comme l'autre, pour des raisons inverses, une absence à l'actuel. Or, ce n'est pas parce que l'événement est passé que la variable $\omega$ devrait s'imposer quantitativement au détriment de la variable $\alpha$. L'énonciateur peut, si telle est sa visée 
d'effet, donner à voir une image sécante du temps d'événement, en cours d'accomplissement, indépendamment de la quantité octroyée à $\alpha$ et $\omega$. En transférant le point I (Instant de conscience actuelle / seuil d'actualité) en I' (Instant de saisie I / seuil d'actualisation), il exerce depuis ce nouveau poste d'observation $^{8}$, logé dans l'immanence du procès, une visée à la fois rétrospective sur la partie déjà accomplie et perspective sur la partie à accomplir. Même si l'événement appartient au révolu (référent expérientiel), il est présenté en cours de résolution, sans garantie aucune sur le devenir de la partie non encore accomplie.

En résumé l'énonciateur peut opérer depuis son présent une projection mentale dans l'intériorité d'un événement passé, présent ou futur dont il n'actualise qu'un seul instant, quelles que soient la localisation de ce seuil d'actualisation dans le temps d'événement ou la situation du temps d'événement dans le temps d'univers. En figuration :

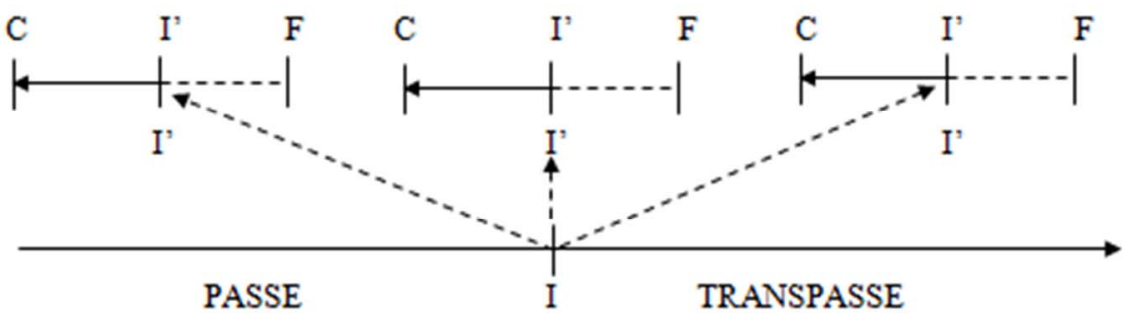

En conclusion, le langage, comme le rappellent d'ailleurs A. Joly et D. O'Kelly, est de l'ordre du «comme si ». BE+ING (re)présente le sujet grammatical (v. supra, § 1.1.) comme s'il était déjà engagé dans le processus événementiel, même si dans le monde réel, le procès est révolu, présent ou futur. L'énonciateur peut choisir (son vouloir dire), dans la disponibilité de ses moyens linguistiques (son savoir dire) de représenter la réalité de telle ou telle façon. Il peut décider, s'il le souhaite, de se projeter mentalement dans l'immanence du procès. L'essentiel pour le locuteur, est de montrer le sujet in medias res, comme s'il était plongé au cœur même de l'action. La localisation de l'instant de saisie sur l'axe du temps d'événement entre $\mathrm{C}$ et $\mathrm{F}$ devient sans importance. De même, la localisation temporelle de l'événement sur l'axe du temps n'a aucune incidence sur la représentation de l'image-temps.

Aussi, l'erreur que commet à mon sens J.-C. Souesme dans son analyse de BE+-ING à « sens futur», est de subir l'ascendant de l'extralinguistique au point de confondre le monde phénoménal avec sa représentation langagière. Dans le passage ci-dessous, l'auteur remet ainsi en cause la position de $\mathrm{P}$. Larreya et $\mathrm{C}$. Rivière qui associent $\mathrm{BE}+\mathrm{ING}$ à l'intériorité du procès :

On nous permettra [...] de ne pas partager leur point de vue selon lequel « la terminaison -ING indique que l'on est à l'intérieur de l'événement en un point quelconque ». [...] comment concilier cette affirmation avec les énoncés à valeur modale pour lesquels «l'événement est situé dans le futur »? (page 38) (1996:136).

Or rien, précisément, n'interdit de (re)présenter le sujet grammatical comme s'il était déjà engagé dans le processus événementiel, ledit événement fût-il situé dans le futur. Comme le souligne avec tant d'àpropos M. Wilmet, «répétons, quitte à lasser le lecteur, que la langue n'est pas un miroir du monde » (1998: 394).

\section{Cas d'exception: saisies sécantes opérées sur un même événement}

Il est pourtant des cas où le principe de variabilité inversement proportionnelle d' $\omega$ et $\alpha$ me paraît s'imposer de lui-même. Il s'applique de manière idoine aux procès grammaticalement imperfectifs référant à un même événement perçu à différents stades de son évolution. L'adaptation de la 
représentation de langue à une représentation de discours me semble alors appropriée car elle se calque sur une logique d'expérience. Il y a congruence entre les trois niveaux de la chronologie notionnelle.

Prenons un exemple. Dans l'avant-dernier chapitre de Vingt mille lieues sous les mers, sans doute l'un des plus sombres de ses romans, Jules Verne décrit la vengeance meurtrière du capitaine Nemo, «ce terrible justicier, véritable archange de la haine ", qui entreprend de couler le cuirassé qui l'a pris en chasse. Mortellement touché par l'éperon d'acier de son adversaire, le cuirassé s'abîme alors lentement et inexorablement vers le fond des océans :

Le capitaine Nemo était là. Muet, sombre, implacable, il regardait par le panneau de bâbord.

Une masse énorme sombrait sous les eaux et, pour ne rien perdre de son agonie, le Nautilus descendait dans l'abîme avec elle. A dix mètres de moi, je vis cette coque entrouverte, où l'eau s'enfonçait avec un bruit de tonnerre, puis la double ligne des canons et les bastingages. Le pont était couvert d'ombres noires qui s'agitaient.

L'eau montait. Les malheureux s'élançaient dans les haubans, s'accrochaient aux mâts, se tordaient sous les eaux. C'était une fourmilière humaine surprise par l'envahissement d'une mer!

Paralysé, raidi par l'angoisse, les cheveux hérissés, l'œil démesurément ouvert, la respiration incomplète, sans souffle, sans voix, je regardais, moi aussi ! Une irrésistible attraction me collait à la vitre !

L'énorme vaisseau s'enfonçait lentement. Le Nautilus, le suivant, épiait tous ses mouvements. Tout à coup, une explosion se produisit. L'air comprimé fit voler les ponts du bâtiment comme si le feu eût pris aux soutes. La poussée des eaux fut telle que le Nautilus dévia.

Alors le malheureux navire s'enfonça plus rapidement (pp. 461-462).

La descente du navire est évoquée à trois reprises différentes, plus ou moins espacées dans le temps : Une masse énorme sombrait sous les eaux / L'énorme vaisseau s'enfonçait lentement / Alors le malheureux navire s'enfonça plus rapidement. L'intérêt de ce passage est qu'il donne à voir l'événement du début à la fin, sous la forme d'un parcours intégral depuis la surface de la mer jusqu'à son lit. C'est parce que la descente du bâtiment est évoquée dans sa totalité qu'il devient possible d'établir un lien logique entre les différents instants de saisie prélevés sur sa progression. Les deux imparfaits saisissent le mouvement à différents stades de son évolution, la part d'inaccompli se résorbant inexorablement en accompli. On voit bien, à travers cet exemple, combien il est important de distinguer la réalité d'expérience de la manière de la (re)présenter. A la mise en relation sur le plan d'expérience peut correspondre alors une mise en relation sur le plan de la représentation. En schéma :

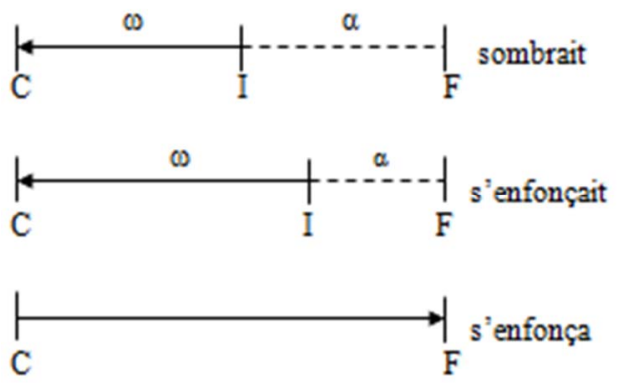

Il est relativement aisé de justifier le passage de l'imparfait au passé simple. Les adverbes alors et plus rapidement introduisent une propriété différentielle susceptible à elle seule de déclencher son utilisation. Ce changement (passage d'une descente lente à une descente rapide) s'inscrit de surcroît dans une succession événementielle régie par des rapports de cause à effet, sous-tendus parfois de relations causales enchâssées (e.g. La poussée des eaux fut telle que le Nautilus dévia). Le premier énoncé (Tout à coup, une explosion se produisit) est donné comme le point de départ de cette réaction en chaîne, le 
dernier énoncé (Alors le malheureux navire s'enfonça plus rapidement), détaché typographiquement dans le texte, comme l'ultime conséquence. Le passé simple installe la chronologie, sans qu'il soit toujours possible de distinguer clairement entre concomitance et consécution événementielle (e.g. L'air comprimé fit voler les ponts du bâtiment / La poussée des eaux fut telle que...).

L'emploi du passé simple appliqué à s'enfoncer a pour effet d'inverser le cinétisme temporel de l'événement désigné jusque là par l'imparfait. De plus, l'adverbe alors, associé à passé simple, confère au procès une valeur nettement inchoative. Seul le premier instant est saisi de manière effective, le reste du parcours perfectif étant impliqué de manière puissancielle. Ce premier instant effectif correspond à l'instant de saisie sécante de l'événement représenté jusque là par l'imparfait. Les deux procès $s$ 'enfonçait et s'enfonça se succèdent sans discontinuité puisqu'il s'agit du même événement, appréhendé successivement de deux manières différentes. Le schéma précédent pourrait donc être modifié comme suit :

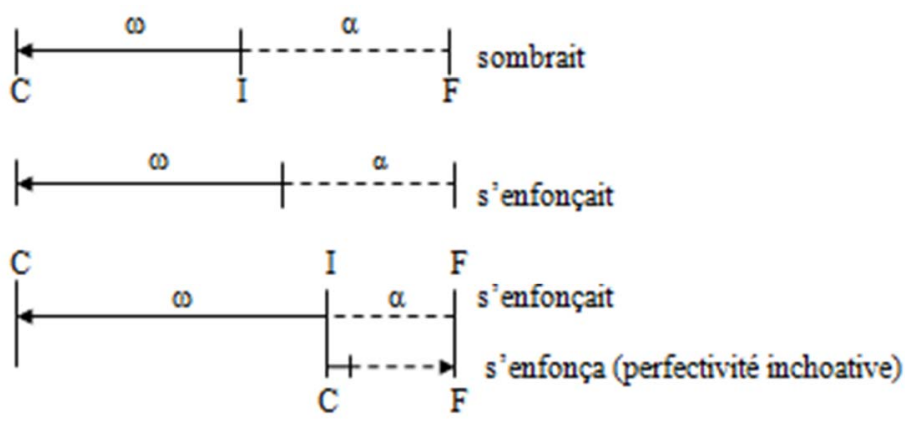

Voici trois traductions proposées pour ces différentes formes verbales :

\begin{tabular}{|l|l|l|l|}
\hline \multicolumn{1}{|c|}{ (a) } & \multicolumn{1}{|c|}{$\begin{array}{c}\text { William Butcher } \\
\text { Traducteur } \\
\text { anonyme } \\
\text { (b) }\end{array}$} & \multicolumn{1}{|c|}{ (c) } \\
\hline $\begin{array}{l}\text { (1) Une masse } \\
\text { énorme sombrait } \\
\text { sous les eaux }\end{array}$ & $\begin{array}{l}\text { An enormous } \\
\text { object was sinking } \\
\text { into the water; }\end{array}$ & $\begin{array}{l}\text { A large mass cast a } \\
\text { shadow on the } \\
\text { water; }\end{array}$ & $\begin{array}{l}\text { The huge mass of } \\
\text { the ship was } \\
\text { sinking. }\end{array}$ \\
\hline $\begin{array}{l}\text { (2) L'énorme } \\
\text { vaisseau } \\
\text { s'enfonçait } \\
\text { lentement. }\end{array}$ & $\begin{array}{l}\text { The enormous } \\
\text { vessel was slowly } \\
\text { sinking. }\end{array}$ & (non traduit) & $\begin{array}{l}\text { The huge ship sank } \\
\text { slowly. }\end{array}$ \\
\hline $\begin{array}{l}\text { (3) Alors le } \\
\text { malheureux navire } \\
\text { s'enfonça plus } \\
\text { rapidement. }\end{array}$ & $\begin{array}{l}\text { Now the unhappy } \\
\text { ship sank more } \\
\text { quickly. }\end{array}$ & $\begin{array}{l}\text { Then the } \\
\text { unfortunate vessel } \\
\text { sank more rapidly. }\end{array}$ & $\begin{array}{l}\text { Then that } \\
\text { unfortunate ship } \\
\text { sank faster. }\end{array}$ \\
\hline
\end{tabular}

Si les deux premières mentions du processus (sombrait et $s$ 'enfonçait) ont donné lieu, en toute logique, à une traduction par le prétérit en BE+-ING, force est de constater que, même en pareil cas, le recours à la forme imperfective n'est pas systématique, ce qui montre que l'on ne peut évoquer que des critères qui favorisent l'emploi de BE+-ING, sans pour autant le contraindre. Par exemple T. Brunetti (3 traducteur) a utilisé le prétérit en BE+-ING pour la première mention (The huge mass of the ship was sinking) mais le prétérit simple pour le deuxième (The huge ship sank slowly). Pourtant, le choix de la forme simple en (2c) est parfaitement légitime. La forme simple sank donne d'ores et déjà une vision globale de l'événement. Même si le procès est saisi en «perfectivité médiane » (traduisible donc par 
l'imparfait ; v. supra, § 2.2.), la limite de fin est en quelque sorte déjà suggérée en pointillés. De plus, la mise en opposition, terme à terme, que permet sank slowly (2c) avec sank faster (3c) montre la fin du processus se profiler en filigrane dans le premier cas (perfectivité médiane $\rightarrow$ imparfait) et se dresser avec la force de l'inéluctable dans le second (perfectivité intégrale $\rightarrow$ passé simple).

\section{Conclusion}

Regroupons les trois principaux cas de figure successivement abordés dans cette étude: l'imparfait narratif (Il y a trois mois un homme bien mis et de grandes manières se présentait chez un bijoutier), la valeur déréalisante de l'imparfait (Un instant après, le train déraillait), et BE+-ING à sens futur (I'm leaving tomorrow). Trois remarques s'imposent : la première est que ces cas, très disparates, donnent lieu à une représentation aspectuelle similaire, avec réduction drastique d' $\omega$ au profit d' $\alpha$, qui occupe alors la quasi-intégralité voire l'intégralité de l'événement. Cette similarité aspectuelle se trouve justifiée par trois raisons fondamentalement différentes : dans le premier cas, l'imparfait se rapproche du passé simple au point de pouvoir commuter avec lui; dans le deuxième, le procès renvoie à un événement contrefactuel; dans le troisième, $\mathrm{BE}+\mathrm{V}-\mathrm{ING}$ réfère à un événement futur. Ces deux derniers critères pouvent être regroupés, si l'on préfère, en un seul, à savoir l'absence à l'actuel. Quoi qu'il en soit, ce sont des causes hétéroclites qui conduisent apparemment au même effet et, pour cette seule raison, on peut se demander s'il est nécessaire de recourir à une représentation de discours. Le souci légitime de proposer une représentation spécifique reflétant le particularisme de l'emploi discursif se voit en quelque sorte contrarié par l'hétérogénéité des cas susceptibles d'être englobés sous une seule et même représentation. La deuxième remarque, plus importante, porte sur la distinction maintes fois opérée dans cette étude entre expérience et représentation. Dès lors que l'on considère que le monde réel et sa représentation langagière sont distincts, il devient, à mon sens, superfétatoire de recourir au principe de déformabilité d' $\omega$ et $\alpha$. La langue est de l'ordre du comme si, elle n'est pas le miroir du monde, tout comme « la carte n'est pas le territoire » (Korzybsky). La troisième remarque porte sur le concept de «décadence transcendante », qui transfère la décadence à l'extérieur du temps d'événement. J'ai souligné les difficultés que générait cette ultime tentative sur le plan théorique. Les variations de l'invariant répondent certes au souci légitime d'une adaptation de la représentation linguistique à la représentation expérientielle. Si le principe n'est pas dénué d'intérêt, loin s'en faut, et s'avère même opératoire dans certains cas, il ne me paraît pas toutefois éclairer systématiquement l'analyse des formes imperfectives du français et de l'anglais.

\section{Bibliographie}

Bardière,Y. (2009), La traduction du passé en anglais et en français. De l'aspect au temps, Paris, L'Harmattan.

Chevalier, J.-C. \& Delport, M.-F. (1995a), « Temps des choses, temps de l'outil », Modèles linguistiques, tome XVI, fasc. 1, vol. 31, pp. 149-164.

Chevalier, J.-C. \& Delport, M.-F. (1995b), L’horlogerie de Saint-Jérôme. Problèmes linguistiques de la traduction, Paris, L’Harmattan.

Chevalier, J.-C. (1999), «L'imparfait narratif: à quel prix?», Cahiers de praxématique, vol. 32, Montpellier, Praxiling, pp. 189-210.

Conrad, J. ([1900] 1957), Lord Jim, Harmondsworth, Penguin Books.

Conrad, J. (1996) Lord Jim, traduit de l'anglais par Odette Lamolle, Paris, Editions Autrement. 
Gosselin, L. (1999), «Le sinistre Fantômas et l'imparfait narratif», Cahiers de praxématique, vol. 32, Montpellier, Praxiling, pp. 19- 42.

Guillaume, G. ([1929] 1965), Temps et verbe. Théorie des aspects, des modes et des temps, Paris, Honoré Champion.

Guillaume, G. ([1964] 1984), Langage et science du langage, Presses de l’Université Laval, Québec ; Paris, Nizet.

Guillaume, G. (1971), Leçons de linguistique 1948-49, série A, vol. 1, Les Presses de 1’Université Laval, Québec, Paris, Klincksieck.

Guillaume, G. (1973), Leçons de linguistique 1948-49, série C, vol. 3, Les Presses de l’Université Laval, Québec, Paris, Klincksieck.

Guillaume, G. (1991), Leçons de linguistique 1943-1944, série A, vol.10, Presses Universitaires de Lille, Les Presses de l’Université Laval, Québec.

Guillaume, G. (1993), Leçons de linguistique, 1938-1939, vol.12, Presses Universitaires de Lille, Les Presses de l’Université Laval, Québec.

Joly, A. \& O’Kelly, D. (1989), L'analyse linguistique des textes anglais, Paris, Nathan.

Joly, A. \& O’Kelly, D. (1990), Grammaire systématique de l'anglais, Paris, Nathan.

Joly, A. (1996), «Les variations d'un "invariant": approche morphogénétique de l'imparfait français», Modèles linguistiques, tome XVII, fasc. 1, vol. 33, pp. 197-202.

Maingueneau, D. (1994), L'énonciation en linguistique française, Paris, Hachette.

Larreya, P. \& Rivière, C. ([1991] 2005), Grammaire explicative de l'anglais, Paris, Pearson Longman.

O’Kelly, D. (1993), «De la Psychomécanique du Langage à la Systématique énonciative» (chapitre 2, en collaboration avec A. Joly), Les théories de la grammaire anglaise en France, Paris, Hachette, pp. 33-62.

O’Kelly, D. (1994), «Du référent expérientiel au référent mental: pour une approche cognitive de la temporalité », Modèles linguistiques, tome XV-1, vol. 29, pp. 25-68.

O’Kelly, D. (2000), « La forme progressive chez les guillaumiens », Cycnos, vol. 17, Université de Nice, pp. 105128.

O'Kelly, D. (2003), «Traduire le temps, traduire l'aspect. Petit précis de systématique comparée» dans Traductologie, linguistique et traduction, Arras, Artois Presses Université, pp. 177-200.

O’Kelly, D. (1998), «Préambule : « Comme si » c'était vrai », Université de Paris-Sorbonne (Paris IV), pp. 239-244.

Pollak, W. (1961), Studien zum «Verbalspekt » im Fransözischen, Vienne, Rohrer.

Pottier, B. (1987), Théorie en analyse linguistique, Paris, Hachette.

Rabatel, A. (2009), Homo narrans, Pour une analyse énonciative et interactionnelle du récit, Tome 1, Limoges, Lambert-Lucas.

Riegel, M. Pellat, J.Ch. \& Rioul, R. ([1994] 1999), Grammaire méthodique du français, Paris, P.U.F.

Souesme, J.-C. (1992), Grammaire anglaise en contexte, Gap, Ophrys.

Tasmowski-De Ryck, L. (1985), « L’imparfait avec et sans rupture », Langue française, 67, pp. 59-77.

Verne, J. ([1870] 1994), Vingt mille lieues sous les mers, Paris, Editions J'ai lu.

Verne, J. (1992), Twenty thousand Leagues under the Sea, traducteur anonyme, Hertfordshire, Wordsworth Editions. 
Verne, J. (1998), Twenty thousand Leagues under the Sea, traduit du français par William Butcher, Oxford University Press.

Verne, J. ([1969] 2001), 20,000 Leagues under the Seas, traduit du français par Mendor T. Brunetti, New York, Signet classic.

Wilmet, M. (1995), «L'articulation mode-temps-aspect dans le système du verbe français », Modèles linguistiques, tome XVI, fasc. 1, vol. 31, pp. 91-110.

Wilmet, M. (1998), Grammaire critique du français, Paris, Bruxelles, Hachette-Duculot.

\footnotetext{
${ }^{1}$ J'ai choisi, dans la représentation graphique, d'orienter le temps d'événement unilatéralement vers la gauche et établir ainsi un parallèle avec BE+-ING (v. § 3.). La question du cinétisme temporel lié à l'imparfait est rarement évoquée.

${ }^{2}$ G. Guillaume utilise cette expression pour désigner la perte notionnelle que peuvent subir les mots, notamment les verbes ayant une vocation à l'auxiliarité. Ainsi, être et avoir, lorsqu'ils sont en position d'auxiliaire, cessent de signifier respectivement « exister » et « posséder ».

${ }^{3}$ Dans le vocabulaire de G. Guillaume, anisogène s'oppose à isogène. Ces deux notions, référant au degré d'homogénéité des noms de discours, peuvent être transposées au domaine verbal. En termes généraux, anisogène désigne «une successivité psychique de deux parties, dont l'une seconde, s'ajoute à la première à titre de renseignement complétif » (Guillaume $1973: 152$ ).

${ }^{4}$ Voir Y. Bardière 2009 pour une analyse contrastive approfondie des phénomènes linguistiques mis en jeu.

${ }^{5}$ Le terme est emprunté à B. Pottier qui en propose la définition suivante : «l'orthonyme est [...] la lexie (mot ou séquence mémorisée) la plus adéquate, sans aucune recherche connotative, pour désigner le référent » $(1987: 45)$.

${ }^{6}$ L'effet « perfectivité », n'est qu'une illusion discursive, un effet d'optique. C'est la raison pour laquelle le terme est mis ici entre guillemets. Quel que soit son emploi, l'imparfait présente un aspect imperfectif.

${ }^{7}$ Autrement dit, si l'événement ne s'est pas produit dans le monde phénoménal, l'accompli se résorbe au profit l'inaccompli qui occupe alors l'entièreté de la configuration aspectuelle. Le concept de variabilité inversement proportionnelle fonctionne aussi dans l'autre sens. Ainsi $\omega=1$ et $\alpha=\varnothing$ dans Le couloir que je quittais ne ressemblait en rien à celui dans lequel je venais d'entrer.

${ }^{8}$ Le concept d'intériorité lié à la saisie sécante l'imparfait a été affiné et modélisé par M.-F. Delport et J.-C. Chevalier. Les auteurs introduisent notamment la notion d' "observateur ", image du locuteur appréhendant l'événement et l'acteur qui y est engagé : «[L'imparfait] permet au locuteur de conférer à la représentation le minimum d'extension requis pour que ce locuteur vienne en observateur prendre pied au ceur de l'événement en train de s'effectuer » (1999: 128-129).

L'observateur renvoie souvent au personnage-énonciateur. A. Rabatel, qui prône également une approche imperfective de l'imparfait, note que c'est Marie qui est le sujet percevant dans Marie entra dans le bureau du président. Il avait l'air épuisé. (2009: 178 sq.). Certes, c'est le locuteur-narrateur qui rapporte la perception, mais c'est l'énonciateur-personnage qui la prend en charge.
} 